\title{
Comparing Avocado, Swamp Bay, and Camphortree as Hosts of Raffaelea lauricola Using a Green Fluorescent Protein (GFP)-Labeled Strain of the Pathogen
}

\author{
A. S. Campbell, R. C. Ploetz, and J. A. Rollins
}

First and second authors: Tropical Research \& Education Center, University of Florida, Homestead 33031; and third author: Plant Pathology, University of Florida, Gainesville 32611.

Current address of first author: U.S. Department of Agriculture-Agricultural Research Service Subtropical Horticulture Research Station, Miami, FL 33158.

Accepted for publication 27 August 2016.

\begin{abstract}
Raffaelea lauricola, a fungal symbiont of the ambrosia beetle Xyleborus glabratus, causes laurel wilt in members of the Lauraceae plant family. North American species in the family, such as avocado (Persea americana) and swamp bay (P. palustris), are particularly susceptible to laurel wilt, whereas the Asian camphortree (Cinnamomum camphora) is relatively tolerant. To determine whether susceptibility is related to pathogen colonization, a green fluorescent protein-labeled strain of $R$. lauricola was generated and used to inoculate avocado, swamp bay, and camphortree. Trees were harvested 3,10, and 30 days after inoculation (DAI), and disease severity was rated on a 1-to-10 scale. By 30 DAI, avocado and

swamp bay developed significantly more severe disease than camphortree (mean severities of 6.8 and 5.5 versus $1.6, P<0.003$ ). The extent of xylem colonization was recorded as the percentage of lumena that were colonized by the pathogen. More xylem was colonized in avocado than camphortree $(0.9 \%$ versus $0.1 \%, P<0.03)$ but colonization in swamp bay $(0.4 \%)$ did not differ significantly from either host. Although there were significant correlations between xylem colonization and laurel wilt severity in avocado $(r=0.74)$, swamp bay $(r=0.82)$, and camphortree $(r=0.87)$, even severely affected trees of all species were scarcely colonized by the pathogen.
\end{abstract}

Raffaelea lauricola (T. C. Harr., Fraedrich \& Aghayeva), a fungal symbiont of the Asian ambrosia beetle Xyleborus glabratus (Eichhoff) (Coleoptera: Curculionidae: Scolytinae), causes laurel wilt in members of the Lauraceae plant family. North American species such as redbay (Persea borbonia (L.) Spreng) and swamp bay (P. palustris (Raf.) Sarg.) are particularly susceptible and have experienced widespread mortality in the southeastern United States (Fraedrich et al. 2008), whereas another American native, avocado (P. americana Mill.), has been recently affected in Florida (Ploetz et al. 2011a, 2013). Camphortree (Cinnamomum camphora (L.) Sieb.), an Asian Lauraceous tree, is more tolerant, as infected trees develop localized symptoms or remain asymptomatic (Fraedrich et al. 2015; Smith et al. 2009). The factors responsible for resistance are not completely understood but disease development may be related to pathogen colonization (Fraedrich et al. 2015).

Symptoms can appear on avocado within weeks of inoculation (Ploetz et al. 2011a). Following inoculation, R. lauricola moves systemically and multiplies in trees, which are killed after defense responses, such as tyloses and gels, obstruct sap flow (Inch et al. 2012). Tylose development was positively correlated with symptom development in avocado (Inch et al. 2012), and response speed may be important in the laurel wilt pathosystem. In other vascular wilt diseases, resistant plants tend to form tyloses abundantly and quickly, preventing vascular wilt pathogens from spreading, whereas susceptible varieties produce few tyloses in advance of pathogen colonization (Pouzoulet et al. 2014; Yadeta and Thomma 2013).

Corresponding author: A. S. Campbell; E-mail address: alina.campbell@ars.usda.gov

(C) 2017 The American Phytopathological Society
Single inoculations with as few as 100 conidia of $R$. lauricola can cause laurel wilt in swamp bay and, to a lesser extent, in avocado (Hughes et al. 2015). In contrast, multiple inoculations are needed to cause branch dieback and wilt symptoms in camphortree (Fraedrich et al. 2015), and some affected trees appear to recover (Cameron et al. 2008).

$R$. lauricola can be isolated from symptomatic tissue on nonselective and selective media and detected using quantitative polymerase chain reaction. However, its visualization in inoculated or diseased tissue has been difficult. Using chitin-specific fluoresceinconjugated wheat germ agglutin (F-WGA) staining and scanning electron microscopy (SEM), Inch et al. (2012) observed a small amount of fungal material in $R$. lauricola-inoculated avocado, although its identity was unclear. Because F-WGA stains fungi nonspecifically, all fungi it stains would fluoresce. And despite the high resolution of SEM, there are insufficient microscopic features that would enable the identification of $R$. lauricola.

To more accurately assess the interaction of $R$. lauricola with avocado, a method to specifically identify the pathogen in infected tissue was needed. Previously, Xiao et al. (2003) transformed the related Ophiostoma piceae with a green fluorescent protein ( $g f p$ ) reporter gene, enabling interactions to be examined between the pathogen and the endophytic fungus, Trichoderma harzianum. Clearly, a GFP-labeled strain of $R$. lauricola would be a valuable tool for examining the laurel wilt pathosystem.

Below is described the production and use of a GFP-labeled strain of $R$. lauricola. Based on relative differences in laurel wilt susceptibility, we hypothesized that the rate and extent of $R$. lauricola colonization would be highest in swamp bay (most susceptible), followed by avocado, then camphortree. Swamp bay, avocado, and camphortree were inoculated with the labeled strain and examined for symptom development, percentage of xylem lumena that were colonized by the pathogen, and the rate of colonization. By 
comparing the timing and extent of colonization among hosts of differing susceptibilities, factors that were correlated with disease development were examined.

\section{MATERIALS AND METHODS}

Transformation, selection, and testing of GFP-labeled R. lauricola. A fungal transformation vector, pCT74 (Lorang et al. 2001), was used to insert a $g f p$ gene into protoplasts of an isolate of $R$. lauricola, CBS 127349, which has been used in previous pathogenicity tests on avocado (Ploetz et al. 2010, 2011a). This vector contains a reengineered $g f p$ sequence, $s g f p$, which is translated more efficiently by filamentous fungi than the wild-type $g f p$ sequence. Sequence $s g f p$ is under the control of the ToxA gene promoter (Ciuffetti et al. 1997) from Pyrenophora tritici-repentis and has been successfully used to express $g f p$ in numerous ascomycetes (Lorang et al. 2001).

Hyphae of $R$. lauricola were harvested from 7-day-old potato dextrose broth cultures, rinsed with water and protoplast buffer, macerated, and incubated on a temperature-controlled shaker for $16 \mathrm{~h}$ at $30^{\circ} \mathrm{C}$ in the presence of Sigma lysing enzymes from T. harzianum ( $200 \mathrm{mg}$ of Sigma enzyme per $3 \mathrm{ml}$ of Novozyme buffer). This yielded $1.415 \times 10^{8}$ protoplasts that were resuspended in $1 \mathrm{ml}$ of protoplast buffer $\left(0.8 \mathrm{M} \mathrm{MgSO}_{4} \cdot 7 \mathrm{H}_{2} \mathrm{O}\right.$ and $0.2 \mathrm{M}$ sodium citrate $\cdot 2 \mathrm{H}_{2} \mathrm{O}$, $\mathrm{pH}$ 5.5).

Escherichia coli DH5 $\alpha$, containing the pCT74 plasmid, was cultured on Luria broth with ampicillin. Plasmid DNA was purified using the Wizard Plus Midipreps DNA Purification System (Promega Corp.), and $4 \mu \mathrm{l}$ (approximately $3 \mu \mathrm{g}$ ) was added to $5 \times$ $10^{6}$ protoplasts in $100 \mu \mathrm{l}$ of protoplast buffer and incubated on ice for $30 \mathrm{~min}$. A polyethylene glycol solution (1 ml) (PEG-3350; Phytotechnologies Laboratory) was added, gently mixed, incubated for $20 \mathrm{~min}$ at room temperature, and spread on a regeneration medium (RM) bottom agar $(0.7 \mathrm{M}$ sucrose, yeast extract at 0.5 $\mathrm{g} /$ liter, and agar at $15 \mathrm{~g} /$ liter) plate. It was incubated overnight at room temperature, and covered with $5 \mathrm{ml}$ of $\mathrm{RM}$ top agar (agar at $15 \mathrm{~g} /$ liter), which contained the selective agent hygromycin B at a final concentration of $300 \mu \mathrm{g} / \mathrm{ml}$ after diffusing through the media.

After 4 days, green fluorescent, regenerating colonies were visible under an epifluorescent Leica DMR microscope (Leica Microsystems Wetzlar GmbH) with a GFP long-pass filter set (450 to $490 \mathrm{~nm}$ excitation). Three vigorously growing colonies were subcultured four times on selective medium (potato dextrose agar [PDA $]+$ hygromycin), one of which was selected and single-spored six times on PDA without selection pressure to ensure transformation stability. Growth rates of the transformed and wild-type strains on half-strength PDA were compared visually over the course of 4 weeks and determined to be similar.

The pathogenicity of the transformed (hereafter, GFP-labeled) strain was confirmed in greenhouse trials at the University of Florida's Tropical Research and Education Center in Homestead, FL, conducted under an FDACS/DPI permit. Healthy plants of the susceptible avocado 'Simmonds' were clonal scions grafted onto seedling rootstocks. They were 4.1 to $6.6 \mathrm{~cm}$ in diameter, and obtained from a local commercial nursery. Plants were inoculated with either a $100-\mu l$ solution of sterile water (control), $1 \times 10^{5}$ conidia of the GFP-labeled strain, or a similar concentration of the wild-type strain, as described previously (Ploetz et al. 2011b). Internal and external symptom development and recovery of the fungus were compared after 3 weeks.

Inoculation study. This experiment was conducted on avocado, swamp bay, and camphortree plants in 12.5 -liter pots. Swamp bay seedlings, 3.6 to $6.6 \mathrm{~cm}$ in diameter, were purchased from a commercial nursery in north Florida; camphortree seedlings, 3.4 to $4.8 \mathrm{~cm}$ in diameter, were transplanted from forested areas surrounding Gainesville, FL; and Simmonds avocado plants were as described above. Trees were maintained at the University of Florida's main campus prior to use and treated with standard fertilizer and irrigation practices.

Trees were inoculated with 3,000 conidia of the GFP-labeled strain, which is toward the higher end of the range of CFU that have been found in a single female of X. glabratus (Campbell 2014; Harrington and Fraedrich 2010). A 2-mm-diameter hole was drilled downward at a $45^{\circ}$ angle in each tree, to a depth of $5 \mathrm{~mm}$. Then $100 \mu$ l of inoculum (sterile water + conidia) or sterile water (control) was pipetted into the hole, and the inoculation site was covered by wrapping the stem with Parafilm.

Trees were harvested 3, 10, and 30 days after inoculation (DAI); then, 30- $\mu \mathrm{m}$ thick cross-sections were taken 5, 20, 50, and $100 \mathrm{~cm}$ above the inoculation point and placed on slides coated with malt agar amended with cycloheximide, streptomycin, ampicillin, and rifampicin (CSMA+) (Harrington 1981; Ploetz et al. 2012), which is somewhat selective for members of the family Ophiostomatales such as $R$. lauricola. After 5 days, the percentage of lumena that were colonized by the pathogen was determined using fluorescence microscopy (Fig. 1). Sections were not surface disinfested.

Three plants of each tree species were sampled at each time point, and the experiment was repeated once; 18 avocado and swamp bay and 16 camphortree plants were used. Due to the absence of observable colonization in camphortree at $3 \mathrm{DAI}$, the 3-day treatment for this species was not repeated in the second experiment; in addition, due to limited availability of camphortree plants of suitable size, the 10-DAI treatment was only repeated using two trees in the second experiment. One control per species was inoculated with sterile deionized water and processed 60 DAI. The experiment was conducted in the Division of Plant Industry's quarantine greenhouse in Gainesville, and tissue was processed and microscopically observed at the University of Florida, Gainesville.

Extent of host colonization and disease development. Host colonization was measured as the percentage of xylem lumena that were colonized, and disease severity was rated on a subjective 1-to-10 scale based on the extent of symptom development $(1=$ healthy to $10=$ dead). Averages of the internal and external severities were used to quantify disease development in individual plants, which were analyzed using mixed models (Proc Mixed); a mean separation table was constructed using differences of least squares means.

A nonparametric analysis of variance (ANOVA) was done on rank-transformed data to compare whether xylem colonization at 3 , 10 , and 30 DAI differed in avocado, swamp bay, and camphortree. Means were separated using Tukey-Kramer comparison lines for least squares means of the species.

A Spearman correlation was run to determine whether disease severity was correlated with $R$. lauricola colonization. Spearman's correlation calculated Pearson's correlation on the ranked values of these data. Ranking (from low to high) means assigned a rank of 1 to the lowest value, 2 to the next lowest, and so on (Altman and Gardner 1988).

Rate of colonization. Rates of colonization in a given crosssection $(5,20,50$, and $100 \mathrm{~cm}$ above the inoculation point) were determined by dividing the distance from the inoculation point (in millimeters) by the number of days after inoculation. A mean rate of colonization was calculated per tree by averaging the millimeters traveled per day by all $R$. lauricola propagules ( 1 propagule $=1$ colonized lumen) observed, or [distance above inoculation point $(\mathrm{mm}) /$ days after inoculation $]=$ rate of colonization .

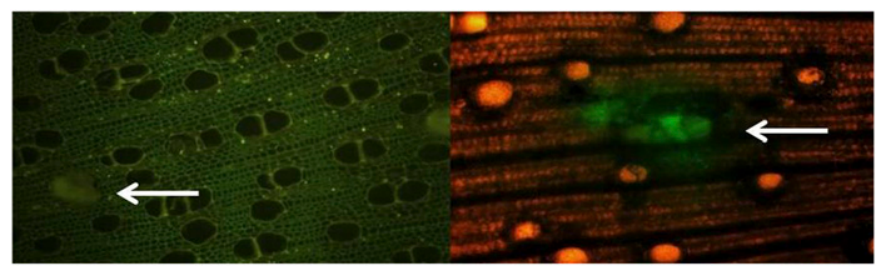

Fig. 1. Green fluorescent protein-labeled Raffaelea lauricola was not detectable immediately after sectioning. It began appearing 3 days after bioenrichment (left, avocado) and was clearly visible after 5 days (right, swamp bay) ( $\times 100$ magnification). White arrows pinpoint colonized lumena. 
A Kruskal Wallis ANOVA was run on rank-transformed data to determine whether rates of colonization at 3,10, and $30 \mathrm{DAI}$ differed among avocado, swamp bay, and camphortree. This nonparametric version of a one-way ANOVA accounted for the nonnormal distribution of these data.

Lumena size. Cross-section photographs at $\times 100$ magnification were used to estimate the largest and smallest diameters of lumena by measuring their size relative to the size of the camera's field of view. Lumena size (in square micrometers) was calculated using the following equation for calculating the area of an ellipse, where $a$ is one-half the largest diameter and $b$ is one-half the smallest diameter of the lumena: area of ellipse (lumena) $=\pi \times a \times b$.

The areas of three lumena (in square micrometers) were calculated at each distance sampled $(5,20,50$, and $100 \mathrm{~cm}$ above the inoculation point), then averaged to determine the mean lumena size for a given tree.

Average lumena size of camphortree, avocado, and swamp bay were analyzed using mixed models (Proc Mixed). A mean separation table was constructed using differences of least squares means.

\section{RESULTS}

Transformation. The $g f p$-transformed strain was stable during serial transfer, fluoresced consistently, and was morphologically indistinguishable from the wild type (data not shown). Transformation and expression of $g f p$ also did not appear to affect the ability to cause laurel wilt on avocado, because trees inoculated with the wild-type and transformed strains developed similar symptoms after 2 weeks and internal disease severities exceeded 5 at 4 weeks after inoculation in each of two preliminary inoculation experiments (data not shown).

The GFP-labeled strain enabled the extent and rate of colonization within a tree to be monitored by visualizing the exact number of colonized lumena in cross-sections. Fluorescent conidial masses began developing 3 days after cross-sections were overlaid onto CSMA (Fig. 1) but were only fully visible 5 days after plating. No fungal structures were visible immediately after sectioning (0 days).

Extent of colonization. At 3 DAI, $R$. lauricola-colonized xylem was not observed in camphortree; however, it was observed in all avocado and swamp bay trees at $5 \mathrm{~cm}$ and in some plants at $20 \mathrm{~cm}$. By 10 DAI, colonized xylem was occasionally observed throughout the entire length of the avocado and swamp bay stems but only as high as $5 \mathrm{~cm}$ in camphortree. Although $R$. lauricola was observed in camphortree plants at the highest point sampled (50 to $100 \mathrm{~cm}$, depending on total tree height) by $30 \mathrm{DAI}$, it was not as evenly distributed throughout the stem as in avocado and swamp bay.

Statistically significant differences in xylem colonization were evident by 30 DAI (Fig. 2). Avocado ( $0.94 \% \pm 0.25$ standard error [SE]) had a significantly higher percentage of colonized xylem than camphortree $0.16 \% \pm 0.1 \mathrm{SE})(F=3.52 \mathrm{df}=15, P<0.03)$ but

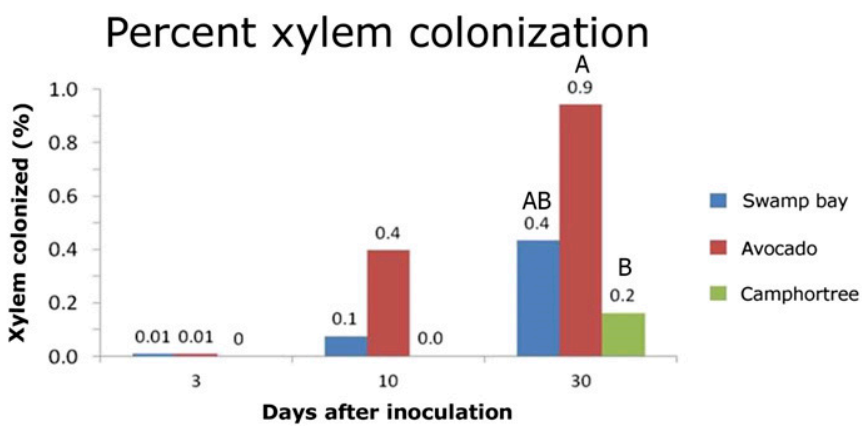

Fig. 2. Percentage of xylem lumena of swamp bay, avocado, and camphortree colonized by a green fluorescent protein-labeled strain of Raffaelea lauricola: Colonization was compared at all days after inoculation but statistically significant differences were only detected at 30 days after inoculation. Means with different letters are significant at $P<0.05$. colonization in swamp bay $(0.43 \% \pm 0.18 \mathrm{SE})$ did not differ significantly from either avocado or camphortree.

Disease development. At $3 \mathrm{DAI}$, internal symptoms were observed only in avocado. After 10 days, external symptoms appeared in avocado and swamp bay, and mild internal symptoms were observed in a single camphortree. By 30 DAI, severe disease developed in avocado and swamp bay but no wilting and only slight defoliation was observed in camphortree. No wilting or internal discoloration was observed in, and the pathogen was not recovered from, water-inoculated control trees.

Differences in disease development among hosts were not significantly different at 3 and 10 DAI but, after 30 days, significantly greater symptoms had developed in avocado $(5.5 \pm 0.8 \mathrm{SE})$ and swamp bay $(6.8 \pm 1.0 \mathrm{SE})$ than in camphortree $(1.8 \pm 0.3 \mathrm{SE})$ $(F=9.73$, Num df $=2$, Den $\mathrm{df}=14, P<0.003$ ) (Fig. 3). However, after 30 days, differences in colonization were only statistically significant between avocado and camphortree (0.94 versus $0.16 \%$ ) (Fig. 2).

Percent xylem colonized and disease ratings (averages of internal and external severities) were significantly and positively correlated in all species. Correlations between colonization and disease severity were very strong in swamp bay $\left(r_{\mathrm{s}}=0.82, P<0.0005\right)$ and camphortree $\left(r_{\mathrm{s}}=0.87, P<0.0005\right)$ and strong in avocado $\left(r_{\mathrm{s}}=0.74\right.$, $P<0.0003$ ), as defined by Swinscow and Campbell (2009). Interestingly, internal symptom development was observed in camphortree plants with as little as $0.001 \%$ of xylem colonized, whereas some avocado and swamp bay plants exhibited no symptoms with up to $0.042 \%$ colonization. The relationship between pathogen colonization and symptom development in different host species warrants further study.

Rate of colonization. Differences in the rates of colonization among avocado, swamp bay, and camphortree were significant at 3 $\mathrm{DAI}\left(\chi^{2}=7.64, \mathrm{df}=2, P<0.02\right)$ and somewhat significant at $10 \mathrm{DAI}$ $\left(\chi^{2}=5.18\right.$, df $\left.=2, P<0.08\right)$, despite the low statistical power of nonparametric tests on small sample sizes (five to six trees per treatment). Although differences in colonization rates at 30 DAI were somewhat significant $\left(\chi^{2}=5.56, \mathrm{df}=2, P<0.06\right)$, these are not considered representative of how quickly $R$. lauricola moved in avocado and swamp bay because the maximum possible distance had been traveled in these hosts (as determined by the height of the tree) prior to that sample date (Table 1).

The maximum mean rates observed for avocado and swamp bay, 26.9 and $32.2 \mathrm{~mm} /$ day, respectively, were at $10 \mathrm{DAI}$, which was only slightly higher than at $3 \mathrm{DAI}$ ( 22.2 and $29.5 \mathrm{~mm} /$ day in avocado and swamp bay, respectively). $R$. lauricola moved more slowly in camphortree, with a maximum rate of $13.7 \mathrm{~mm} /$ day, roughly half that in avocado and swamp bay.

Lumen area. The sizes of lumena in avocado, swamp bay, and camphortree were significantly different $(F=21.27$, df $=24$,

\section{Average disease severity rating}

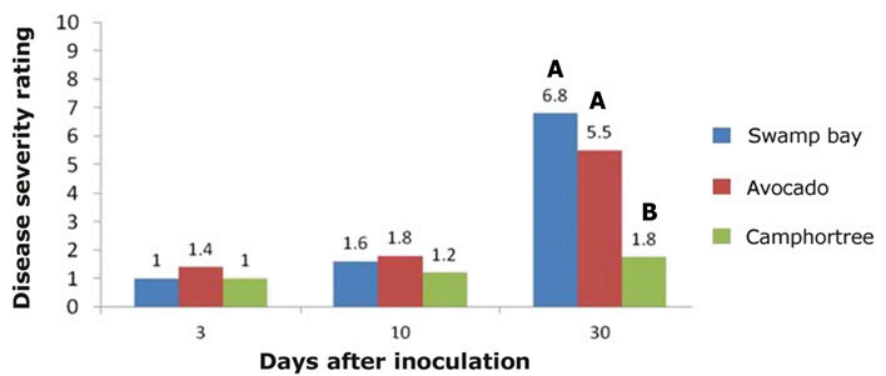

Fig. 3. Average disease severity ratings in swamp bay, avocado, and camphortree after inoculation with green fluorescent protein-labeled Raffaelea lauricola: more disease developed in swamp bay and avocado than camphortree after 30 days. Disease ratings were compared at all days after inoculation but statistically significant differences were only detected at 30 days after inoculation. Means with different letters are significant at $P<0.05$. 
$P<0.0001)$. Avocado lumena $\left(3,063 \pm 226 \mu \mathrm{m}^{2}\right)$ were nearly 2.5 times larger than those in camphortree $\left(1,250 \pm 221 \mu^{2}\right)$, whereas swamp bay lumena were of an intermediate size $\left(2,052 \pm 113 \mu \mathrm{m}^{2}\right)$ (Fig. 4).

\section{DISCUSSION}

Unlike swamp bay and avocado, which are indigenous to North America, camphortree evolved in the same region as X. glabratus and $R$. lauricola. The extents to which naïve American hosts are susceptible and putatively coevolved hosts from Asia are resistant to laurel wilt, as well as host attributes that are associated with these responses, warrant further study (Ploetz et al. 2013).

With the GFP-labeled strain of $R$. lauricola, it was possible to measure the percentage of xylem that was colonized by the pathogen. It enabled study of the spatial distribution of the pathogen, even though visualization depended on a bioenrichment step. Xiao et al. (2003) were also unable to observe their GFP-labeled strain of $O$. piceae immediately after sectioning wood. It is unclear whether the labeled strain of $R$. lauricola needs to be actively growing in order to be visualized, whether thin sectioning damaged the cells of the transformed strain, or whether aggregations of $R$. lauricola propagules are small and, thus, unlikely to be observed microscopically in individual sections.

As hypothesized, the degree of host susceptibility to laurel wilt was associated with the extent of xylem colonization by $R$. lauricola. In both colonization and symptom development, significant differences were found only at 30 DAI, suggesting that colonization and symptom development are temporally close. Camphortree developed significantly less disease than avocado and swamp bay, and correspondingly less xylem was colonized in this host. Although the most severe disease developed in swamp bay 30 DAI, differences in xylem colonization in swamp bay and camphortree were only significant at $P<0.14$. This may be due to the small sample sizes used $(n=6)$. Nonetheless, even the most severely affected swamp bay trees had very low levels of colonization $(\leq 1.6 \%)$.

Small amounts of xylem (up to $0.042 \%$ ) were colonized in avocado and swamp bay trees in the absence of visible symptoms. In contrast, sapwood discoloration was observed in camphortree when as little as $0.001 \%$ of the xylem was colonized. Camphortree plants with relatively high percentages of colonization lost leaves but did not wilt. The sensitive response of this species to $R$. lauricola (i.e., defoliation and sapwood discoloration but very few colonized lumena) may protect it from greater disease development compared with avocado and swamp bay. For example, greater sapwood discoloration in camphortree may indicate a greater production of defense compounds, such as reactive oxygen species, by the host. Tylose development was positively correlated with symptom development in avocado (Inch et al. 2012). Information is needed on the roles that tylose (speed and extent) and secondary metabolite formation play in host response to this disease.

The present study confirmed the minor presence of $R$. lauricola that was noted in susceptible hosts in previous work (Hughes et al. 2015; Inch et al. 2012), and indicates that the pathogen spreads and propagates to an even lower extent in the tolerant host, camphortree. The fact that numerous attacks by $X$. glabratus were needed for symptoms to develop in camphortree (Fraedrich et al. 2015) appears to support the observation that higher amounts of $R$. lauricola are needed to cause symptoms in this host. However, it is not clear what attributes are associated with either pathogen containment or reduced growth in this or other host trees. Research is warranted on whether resistance is a combination of rapid response to $R$. lauricola, anatomical characteristics of host trees, or other characteristics (e.g., chemical constituents) in trees that make them less amenable to R. lauricola multiplication or spread. Regardless of susceptibility, the extent of xylem colonization by the pathogen was strongly correlated with symptom development in all hosts tested.

Over 30 different compounds have been found in camphortree xylem, many of which have antifungal and antimicrobial activity $(\mathrm{Li}$ et al. 2014). Maner et al. (2012) recovered fewer $R$. lauricola spores from the surface of camphortree than redbay blocks 3 weeks after inoculation, suggesting that it is a less suitable substrate for this fungus.

Lumen size varies within a tree based on the stage of tissue development and the tree's size (England and Attiwill 2007). We propose that the smaller lumena we observed in camphortree, versus avocado and swamp bay, may hinder the movement of $R$. lauricola in this host. Clearly, the ability of host plants to contain vascular pathogens is a critical determinant in their ability to resist the diseases they cause. Xylem characteristics that slow the rate of pathogen spread and increase the speed at which vessels are blocked should enhance disease resistance; narrow vessels could be more easily sealed to contain a pathogen, whereas shorter vessels would present the pathogen with more obstacles to movement, such as xylem plates and pit membranes (Pouzoulet et al. 2014). To date, these relationships are best understood in the Dutch elm disease pathosystem, in which resistant trees have narrower and shorter vessels (Miller and Elgersma 1976).

Although Ploetz et al. (2015) did not evaluate xylem dimensions, they observed significantly greater sap flow in a laurel wiltsusceptible avocado cultivar, prior to inoculation with $R$. lauricola, when compared with two moderately susceptible cultivars. Studies

\section{Lumena area by species}

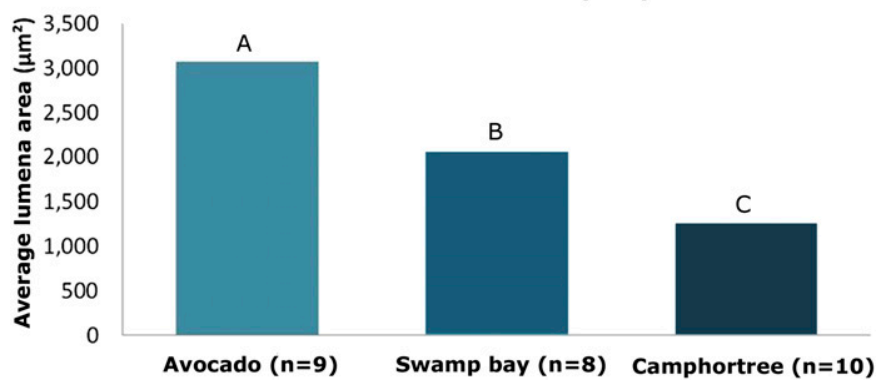

Fig. 4. Mean lumena size of avocado, swamp bay, and camphortree trees. In all, 9 to 15 lumena were measured per tree. Means followed by different letters are significantly different at $P<0.05$.

TABLE 1. Mean rate of Raffaelea lauricola colonization per tree following artificial inoculation ${ }^{\mathrm{x}}$

\begin{tabular}{|c|c|c|c|c|}
\hline \multirow[b]{2}{*}{ Time of test ${ }^{\mathrm{z}}$} & \multicolumn{3}{|c|}{ Average speed $( \pm \mathrm{SE})(\mathrm{mm} / \text { day })^{\mathrm{y}}$} & \multirow[b]{2}{*}{$P$ value } \\
\hline & Avocado & Swamp bay & Camphortree & \\
\hline 3 DAI & $22.2( \pm 9.3)(n=6) \mathrm{A}$ & $29.5( \pm 11.0)(n=5) \mathrm{A}$ & $0( \pm 0)(n=5) \mathrm{B}$ & $<0.02$ \\
\hline 30 DAI & $8.4( \pm 1.0)(n=6)$ & $13.5( \pm 2.5)(n=6)$ & $13.7( \pm 3.0)(n=6)$ & $<0.06$ \\
\hline
\end{tabular}

${ }_{\mathrm{x}}$ A Kruskal Wallis analysis of variance was run on rank-transformed data to determine whether rates of colonization differed among avocado, swamp bay, and camphortree. Means followed by the same letter within columns are not significantly different at $P<0.05$.

y Mean rate of colonization was calculated per tree by averaging the millimeters/day traveled by all $R$. lauricola propagules observed in cross-sections (see text); $n=$ number of trees tested and $\mathrm{SE}=$ standard error.

${ }^{\mathrm{z}}$ Colonization rates differed significantly at 3 days after inoculation (DAI) $(P<0.02)$. 
are underway to more fully understand xylem attributes that are associated with the response of avocado to this pathogen.

From this study, it can be inferred that differences in susceptibility were related to pathogen colonization; however, it is not clear what causes these differences. Resistance to laurel wilt was associated with less xylem colonization in camphortree versus avocado and swamp bay but little xylem colonization was required for disease to develop in any of these hosts. Following inoculation, $R$. lauricola moved more quickly in avocado and swamp bay than in camphortree. Additional research is needed to determine whether physiological or anatomical attributes of these hosts are responsible for these differences and the ultimate extent to which they develop symptoms of laurel wilt.

\section{ACKNOWLEDGMENTS}

This research was partially funded by United States Department of Agriculture National Institute of Food and Agriculture grant 2009-5118105915 to R. C. Ploetz. We thank A. Black for propagating camphortree plants that were used in these studies, and J. Colee (University of Florida Institute of Food and Agricultural Sciences Statistics Department) for help with the statistical analysis.

\section{LITERATURE CITED}

Altman, D. G., and Gardner, M. J. 1988. Statistics in medicine: Calculating confidence intervals for regression and correlation. Br. Med. J. (Clin. Res. Educ.) 296:1238.

Cameron, R. S., Bates, C., and Johnson, J. 2008. Distribution and spread of laurel wilt disease in Georgia: 2006-08 Survey and field observations. Online publication. Georgia Forestry Commission. http://fhm.fs.fed.us/em/ funded/09/so-em-08-02-report.pdf

Campbell, A. 2014: Characterizing pathogen-vector-host interactions in laurel wilt, a disease of avocado. Ph.D. dissertation, University of Florida.

Ciuffetti, L. M., Tuori, R. P., and Gaventa, J. M. 1997. A single gene encodes a selective toxin causal to the development of tan spot of wheat. Plant Cell 9: 135-144.

England, J. R., and Attiwill, P. M. 2007. Changes in sapwood permeability and anatomy with tree age and height in the broad-leaved evergreen species Eucalyptus regnans. Tree Physiol. 27:1113-1124.

Fraedrich, S. W., Harrington, T. C., and Best, G. S. 2015. Xyleborus glabratus attacks and systemic colonization by Raffaelea lauricola associated with dieback of Cinnamomum camphora in the southeastern United States. For. Pathol. 45:60-70.

Fraedrich, S. W., Harrington, T. C., Rabaglia, R. J., Ulyshen, M. D., Mayfield, A. E., III, Hanula, J. L., Eickwort, J. M., and Miller, D. R. 2008. A fungal symbiont of the redbay ambrosia beetle causes a lethal wilt in redbay and other Lauraceae in the southeastern USA. Plant Dis. 92:215-224.

Harrington, T. C., and Fraedrich, S. W. 2010. Quantification of propagules of the laurel wilt fungus and other mycangial fungi from the redbay ambrosia beetle, Xyleborus glabratus. Phytopathology 100:1118-1123.

Harrington, T. C. 1981. Cycloheximide sensitivity as a taxonomic character in Ceratocystis. Mycologia 73:1123-1129.

Hughes, M. A., Inch, S. A., Ploetz, R. C., Er, H. L., Bruggen, A. H. C., and Smith, J. A. 2015. Responses of swamp bay, Persea palustris, and avocado,
Persea americana, to various concentrations of the laurel wilt pathogen, Raffaelea lauricola. For. Pathol. 45:111-119.

Inch, S., Ploetz, R., Held, B., and Blanchette, R. 2012. Histological and anatomical responses in avocado, Persea americana, induced by the vascular wilt pathogen, Raffaelea lauricola. Botany 90:627-635.

Li, Q., Wang, X. X., Lin, J. G., Liu, J., Jiang, M. S., and Chu, L. X. 2014. Chemical Composition and Antifungal Activity of Extracts from the Xylem of Cinnamomum camphora. BioResources 9:2560-2571.

Lorang, J. M., Tuori, R. P., Martinez, J. P., Sawyer, T. L., Redman, R. S., Rollins, J. A., Wolpert, T. J., Johnson, K. B., Rodriguez, R. J., Dickmann, M. B., and Ciufetti, L. M. 2001. Green fluorescent protein is lighting up fungal biology. Appl. Environ. Microbiol. 67:1987-1994.

Maner, M. L., Fraedrich, S., and Hanula, J. L. 2012. Growth of the laurel wilt pathogen Raffaelea lauricola, on wood of various tree species. M.S. thesis, University of Georgia.

Miller, H. J., and Elgersma, D. M. 1976. The growth of aggressive and nonaggressive strains of Ophiostoma ulmi in susceptible and resistant elms, a scanning electron microscopical study. Neth. J. Plant Pathol. 82:51-65.

Ploetz, R. C., Harrington, T., Hulcr, J., Bostock, R., Eskalen, A., Farber, B., Crane, J., Harmon, C., Inch, S., Palmateer, A., Peña, J., Smith, J., Kendra, P., Schnell, R., Fraedrich, S., Hanula, J., Mayfield, A., Rabaglia, R., and Wingfield, M. 2011a. Recovery Plan for Laurel Wilt of Avocado. National Plant Disease Recovery System. Homeland Security Presidential Directive Number 9 (HSPD-9). Online publication. http://www.ars.usda.gov/ SP2UserFiles/Place/00000000/opmp/Avocado\%20LW\%20110829.pdf

Ploetz, R. C., Hulcr, J., Wingfield, M. J., and De Beer, Z. W. 2013. Destructive tree diseases associated with ambrosia and bark beetles: Black swan events in tree pathology. Plant Dis. 97:856-872.

Ploetz, R. C., Pérez-Martínez, J. M., Evans, E. A., and Inch, S. A. 2011b. Towards fungicidal management of laurel wilt of avocado. Plant Dis. 95:977-982.

Ploetz, R. C., Pérez-Martínez, J. M., Smith, J. A., and Hughes, M. 2010. Laurel wilt of avocado: Assessing genotypic responses, factors that influence disease development, and the potential for management with fungicides. Int. For. Rev. 12:398.

Ploetz, R. C., Pérez-Martínez, J. M., Smith, J. A., Hughes, M., Dreaden, T. J., Inch, S. A., and Fu, Y. 2012. Responses of avocado to laurel wilt, caused by Raffaelea lauricola. Plant Pathol. 61:801-808.

Ploetz, R. C., Schaffer, B., Vargas, A. I., Konkol, J. L., Salvatierra, J., and Wideman, R. 2015. Impact of laurel wilt, caused by Raffaelea lauricola, on leaf gas exchange and xylem sap flow in avocado, Persea americana. Phytopathology 105:433-440.

Pouzoulet, J., Pivovaroff, A., Santiago, L., and Rolshausen, P. E. 2014. Can vessel dimension explain tolerance toward fungal vascular wilt diseases in woody plants? Lessons from Dutch elm disease and esca disease in grapevine. Front. Plant Sci. 5:253.

Smith, J. A., Mount, L., Mayfield, A. E., III, Bates, C. A., Lamborn, W. A., and Fraedrich, S. W. 2009. First report of laurel wilt disease caused by Raffaelea lauricola on camphor in Florida and Georgia. Plant Dis. 93:198.

Swinscow, T. V., and Campbell, M. J. 2009. Correlation and regression. Pages 119-132 in: Statistics at Square One, 11th ed. BMJ Books, London.

Xiao, Y., Ciuffetti, L. M., and Morrell, J. J. 2003. Transformation of Ophiostoma piceae and Trichoderma harzianum with a green fluorescent protein (GFP) gene. In: 34th Annu. Meet. Int. Res. Group Wood Preserv. Document IRG/WP'03-10477. IRG Secretariat, SE-100 44 Stockholm, Sweden.

Yadeta, K. A., and Thomma, B. P. 2013. The xylem as battleground for plant hosts and vascular wilt pathogens. Front. Plant Sci. 4:97. 